\title{
Brain symmetry plane computation in MR images using inertia axes and optimization
}

\author{
Alexander V. Tuzikov ${ }^{1}$, Olivier Colliot ${ }^{2}$ and Isabelle Bloch ${ }^{2}$ \\ ${ }^{1}$ Institute of Engineering Cybernetics \\ Academy of Sciences of Republic Belarus \\ Surganova 6, 220012 Minsk, Belarus \\ tuzikov@mpen.bas-net.by \\ ${ }^{2}$ Ecole Nationale Supérieure des Télécommunications \\ Département TSI, CNRS URA 820 \\ 46 rue Barrault, 75634 Paris Cedex 13, France \\ \{Olivier.Colliot,Isabelle.Bloch\}@enst.fr
}

\begin{abstract}
Detection of the best symmetry plane in $3 D$ images can be treated as a registration problem between the original and the reflected images. The registration is performed in a $3 D$ space of parameters defining orientation and shift of a reflection plane. We use the normalized $l_{2}$ metric as the similarity measure between original and reflected images and investigate an algorithm for computation of the best symmetry plane. The algorithm computes first an initial position of the plane by analyzing principal inertia axes. We demonstrate on several MR brain images that the initial position is in the neighborhood of the global maximum. Therefore the downhill simplex method is further used for the computation of the best symmetry plane. The proposed algorithm was tested on simulated and real MR brain images.

Keywords: Symmetry plane, symmetry measure, MR brain image, mid-sagittal plane, inertia axes, optimization
\end{abstract}

\section{Introduction}

The mid-sagittal plane is defined as the plane separating both brain hemispheres. The automatic detection of this plane in brain images is a useful task. It can be used to reorient images, for example for further alignment in the Talairach reference frame [7]. It can also be a first step in intra-subject registration [2] or serve as a basis for the study of dissymetries in the brain [17].

Brain images possess a high degree of plane symmetry although they are not exactly symmetrical. Medical studies (see, for example, [1] for some corresponding references) demonstrate brain anatomical asymmetries in sizes of the frontal and occipital lobes, in surface of the planum temporale and planum parietale, in the anterior speech region. The plane with respect to which the brain is the most symmetrical is called the brain symmetry plane. This plane is often considered as the first approximation of the mid-sagittal plane [5]. The main goal of this paper is to develop an algorithm for the symmetry plane computation in MR brain images.

There is a vast literature in mathematics, image processing and computer vision domains dealing with different kinds of symmetry (central, reflection, rotation, skew) of shapes and images. Let us point out, for example, some publications $[10,11]$ devoted to the reflection symmetry. Most of practical algorithms developed for symmetry measurements are applied in the 2D case and only some of them can be extended to 3D [18]. Principal axes of inertia were used in [13] to define the best plane of reflection symmetry. An algorithm for finding symmetry planes of 3D objects using extended Gaussian image representation was developed in [16]. Octree representation of 3D objects is used in [12] to compute their symmetry degree. Recently several new algorithms were proposed for symmetry plane computation in 3D images of the brain $[2,7,15,17]$.

The problem of finding symmetry plane and reflection symmetry measure can be treated as a rigid registration problem between the original and the reflected images. Therefore, one can use different similarity measures known in the literature [8] (for example, correlation coefficient, correlation ratio, mutual information). Since the problem belongs to the class of monomodal registration, then the cor- 
relation coefficient or $l_{2}$ metric seem to be the most suitable similarity measures.

A method for computation of the brain symmetry plane using optimization technique is presented in [2]. A number of plane positions equally distributed in the space of plane orientations are used there as candidates for finding the initial plane position for the optimization procedure. We propose (Section 3) an algorithm that uses a single initial plane for the further optimization. This plane is computed from the ellipsoid of inertia and we show in Section 4 for MR brain images that it belongs to the region of the global maximum of the similarity measure under consideration. Therefore, starting the optimization from this plane gives more chances to achieve the global maximum and besides reduces substantially the complexity of the algorithm.

\section{Symmetry measure definition}

Let $\mathbf{u}$ be a unit vector in $\mathbb{R}^{3}, S^{2}$ is the unit sphere of all possible directions in $\mathbb{R}^{3}$, and $\Pi_{\mathbf{u}, d}$ is a plane in $\mathbb{R}^{3}$ orthogonal to the vector $\mathbf{u}$ and passing at the distance $d$ from the coordinate origin. We denote by $e_{\mathbf{u}, d}(f)$ the reflection of image $f$ with respect to the plane $\Pi_{\mathbf{u}, d}$ :

$$
e_{\mathbf{u}, d}(f)(x, y, z)=f\left(e_{\mathbf{u}, d}(x, y, z)\right) .
$$

An image $f$ is called reflection symmetrical if there exists a reflection plane $\Pi_{\mathbf{u}, d}$ such that $e_{\mathbf{u}, d}(f)=f$. We say in this case that $\Pi_{\mathbf{u}, d}$ is a plane of reflection symmetry for $f$.

Suppose that one is able to compute the symmetry measure $\mu_{\mathbf{u}, d}(f)$ of the image $f$ with respect to an arbitrary reflection plane $\Pi_{\mathbf{u}, d}$. Then the problem we are dealing with in this paper is to find the plane of maximal symmetry degree of image $f$ and the corresponding value of symmetry measure $\mu(f)$ :

$$
\mu(f)=\max _{\mathbf{u} \in S^{2}, d \in \mathbb{R}^{+}} \mu_{\mathbf{u}, d}(f) .
$$

When comparing images, it is usually assumed that a distance or a similarity measure $\sigma\left(f_{1}, f_{2}\right)$ is defined on the set of images. In this case the symmetry measure $\mu_{\mathbf{u}, d}(f)$ can be defined as the similarity between images $f$ and $e_{\mathbf{u}, d}(f)$, i.e. $\mu_{\mathbf{u}, d}(f)=\sigma\left(f, e_{\mathbf{u}, d}(f)\right)$.

Different similarity measures are known in the literature for comparing images. In this report we use $l_{2}$ metric $\left(l_{2}(f, g)=\|f-g\|\right)$ and the following symmetry measure:

$$
\mu_{\mathbf{u}, d}(f)=1-\frac{\left\|f-e_{\mathbf{u}, d}(f)\right\|^{2}}{2\|f\|^{2}}
$$

\section{Symmetry plane computation}

To compute the reflection symmetry plane one needs to check all possible orientations and translations of the reflec- tion plane. It is an optimization problem in 3 dimensional parametric space (here $\beta$ and $\alpha$ are the angles of the plane normal vector with the $x$ axis and $x y$ plane, respectively, and $d$ is the distance to the plane from the coordinate origin). This computation is time consuming. Therefore it seems useful to check first the most appropriate positions of reflection planes. These positions can be used as initial points for the further optimization.

The initial positions can be interpreted, for example, in terms of ellipsoid of inertia [4]. The axes of the ellipsoid of inertia for a 3D body are defined by the eigenvectors of the corresponding covariation matrix.

It is known that if a body possesses a plane reflection symmetry then the symmetry plane passes through its center of mass and is orthogonal to some ellipsoid axis. Therefore for computation of a reflection symmetry measure in this case it is sufficient to check 3 planes only.

Suppose that the center of mass of the ellipsoid of inertia of image $f$ is $\mathbf{b}_{c}=\left(x_{c}, y_{c}, z_{c}\right)$ and the eigenvectors are $\mathbf{u}_{1}, \mathbf{u}_{2}, \mathbf{u}_{3}$. Then one can compute

$$
\max \left\{\mu_{\mathbf{u}_{1}, \mathbf{u}_{1} \cdot \mathbf{b}_{c}}(f), \mu_{\mathbf{u}_{2}, \mathbf{u}_{2} \cdot \mathbf{b}_{c}}(f), \mu_{\mathbf{u}_{3}, \mathbf{u}_{3} \cdot \mathbf{b}_{c}}(f)\right\},
$$

where $\mathbf{u} \cdot \mathbf{b}$ denotes the inner product of vectors $\mathbf{u}$ and $\mathbf{b}$.

The best position of the reflection symmetry plane found from the ellipsoid of inertia can be further improved using optimization in the $3 \mathrm{D}$ space of reflection plane parameters. In our implementation we used the downhill simplex method [14]. Since the downhill simplex method is a local optimization method there is no guaranty in general of finding the global maximum. However, in Section 4 we demonstrate experimentally that one can obtain good solutions for MR brain images using this approach.

\section{Algorithm 1}

1. Computation of the initial symmetry plane from the ellipsoid of inertia.

2. Improving the orientation and translation of the symmetry plane and the symmetry measure computation using the optimization technique.

\section{Experiment discussion}

We investigated the graphs of $\mu_{\mathbf{u}, d}(f)$ for different values of $\mathbf{u}$ and $d$ for 5 real MR normal brain images. These graphs have a similar form (see Fig. 1). Fig. 1(a) shows the profile of $\mu_{\mathbf{u}, d}(f)$ for the brain image presented in Fig. 2 for reflection planes passing through the image center of mass. The function values are computed for angles $\alpha, \beta \in[-\pi / 2, \pi / 2)$ with an incremental step of 0.05 radian. The function graph has a regular form with a global 
maximum in the neighborhood of the initial point. One can see in Fig. 1(c) that the initial position is in the neighborhood of the global maximum (here + and $*$ indicate the initial and final orientation of the reflection plane obtained after the optimization step). Fig. 1(b) shows the profile of $\mu_{\mathbf{u}, d}(f)$ for angles $\alpha, \beta \in[0,0.1]$ (in radians) and a neighborhood of the center of mass $d \in\left[d_{c}-5, d_{c}+5\right]$. It means that for every orientation of the reflection plane in the neighborhood of the global maximum the symmetry measure decreases while shifting the plane away from the center of mass. To simplify the visualization, every pair $(\alpha, \beta)$ is shown as one point on the axis. Summarizing we can conclude that in the tested MR brain images the initial position of the plane obtained from the ellipsoid of inertia belongs to the region of global maximum of the symmetry measure. It allows to go up to the function global maximum using the local optimization technique.

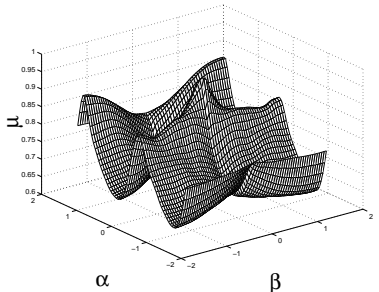

(a)

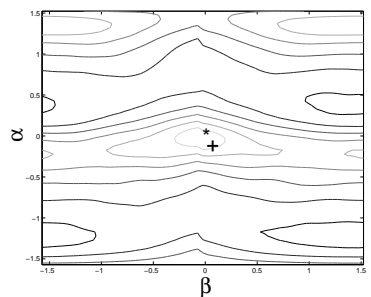

(c)

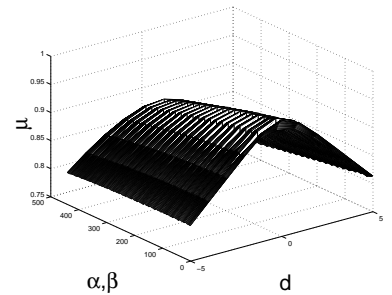

(b)
Figure 1. The graphs of function $\mu_{\mathbf{u}, d}(f)$ computed for different values of $\mathrm{u}$ and $d$.

Algorithm 1 was tested on a public available 3-D simulated brain database from the McConnell Brain Imaging Center at McGill University (http://www.bic.mni.mcgill.ca/brainweb/). The normal brain database contains simulated brain MR images for three modalities (T1-, T2-, and PD(proton-density)weighted) and a variety of slice thicknesses, noise levels, and levels of intensity non-uniformity [3, 6]. We tested the algorithm for 54 images for each modality (with 3 slice thicknesses 1,3,5 mm, 6 levels of noise $0 \%, 1 \%, 3 \%, 5 \%, 7 \%, 9 \%$ and 3 levels of intensity nonuniformity $0 \%, 20 \%, 40 \%$ ). An additional advantage of using this database for symmetry analysis is that the images are aligned in the stereotactic space and the mid-sagittal plane coincides with $y z$ plane. For all these images the orientation of the best symmetry plane found by Algorithm 1 coincides with the mid-sagittal plane. The maximal absolute difference $m d(|\alpha|)$ for $\alpha$ is 0.42 degree, the mean absolute value $m v(|\alpha|)=0.16$ degree and the standard deviation $\operatorname{std}(|\alpha|)=0.13$ degree. The values for $\beta$ are $m d(|\beta|)=0.58, m v(|\beta|)=0.16$ and $\operatorname{std}(|\beta|)=0.08$ degrees, respectively. This confirms that the proposed algorithm is stable in the presence of noise and image non-uniformity.

Algorithm 1 was also tested for 24 real MR normal brain images (in T1 modality) and for all of them we obtained good final results (see an example in Fig. 2). For 19 images we applied the process directly on original images (without any pre-processing or segmentation) and obtained good final results (see third and fourth rows of Fig. 2). For 5 others we compared the position of the symmetry plane found for the original image and for the corresponding segmented brain. The segmentation of the brain was obtained by a 3D mathematical morphology algorithm [9]. The final results were similar. The mean value and the standard deviation of absolute differences for $\alpha$ and $\beta$ were $m v\left(\left|\alpha_{1}-\alpha_{2}\right|\right)=0.5, \operatorname{std}\left(\left|\alpha_{1}-\alpha_{2}\right|\right)=0.39$ and $m v\left(\left|\beta_{1}-\beta_{2}\right|\right)=0.76$, std $\left(\left|\beta_{1}-\beta_{2}\right|\right)=0.73 \mathrm{de}-$ grees respectively. Here indices 1 and 2 correspond to original and segmented brain images. Therefore, it seems that the algorithm can be applied successfully for original MR images. For 4 images we compared the results obtained for a binary mask of the segmented brain and for the gray-level image itself. The final results were also simi$\operatorname{lar}\left(m v\left(\left|\alpha_{2}-\alpha_{3}\right|\right)=0.59\right.$, std $\left(\left|\alpha_{2}-\alpha_{3}\right|\right)=0.46$ and $m v\left(\left|\beta_{2}-\beta_{3}\right|\right)=0.61, \operatorname{std}\left(\left|\beta_{2}-\beta_{3}\right|\right)=0.58$ degrees assuming that index 3 corresponds to a brain mask image). It means that the brain shape defines the position of the symmetry plane with a high precision.

\section{Conclusion}

We proposed an algorithm for computation of the best symmetry plane and the plane symmetry measure for 3D images. The initial position of the plane is computed from the corresponding ellipsoid of inertia. This position is further improved using the downhill simplex method. This algorithm can be applied for MR brain images. We demonstrated on several simulated and real MR brain images that the initial position is in the neighborhood of the symmetry measure global maximum. Therefore a local optimization method allows to find efficiently the global maximum.

It was shown on synthetic MR brain images that the algorithm is stable to noise and intensity non-uniformity. Further work includes comparing the method to other approaches and studying its behavior in pathological cases.

A. Tuzikov was supported by a sabbatical grant at the 

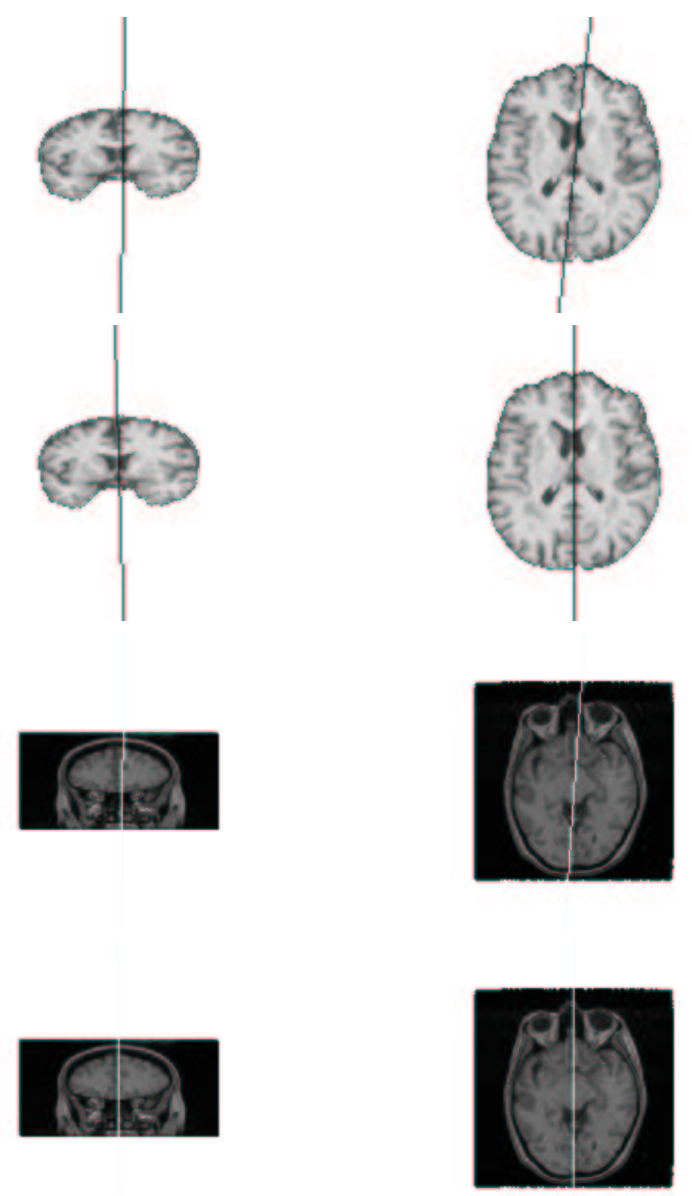

Figure 2. Coronal and axial slices of the segmented and non-segmented brain images: (first row) initial symmetry plane computed from the ellipsoid of inertia; (second row) the best symmetry plane found by Algorithm 1; (third row) initial plane; (fourth row) the best symmetry plane.

Department of Signal and Image Processing (ENST) and is very grateful to the department for its hospitality. We would like to thank CHU La Pitié-Salpétrière (Paris), CHU La Timone (Marseille), INSERM 280 and Hôpital Neurologique (Lyon) for providing the images.

\section{References}

[1] K. Amunts, L. Jäncke, H. Mohlberg, H. Steinmetz, and K. Zilles. Interhemispheric asymmetry of the human motor cortex related to handedness and gender. Neuropsychologia, 38:304-312, 2000.

[2] B. Ardekani, J. Kershaw, M. Braun, and I. Kanno. Automatic detection of the mid-sagittal plane in $3 \mathrm{D}$ brain im- ages. IEEE Transactions on Medical Imaging, 16(6):947952, 1997.

[3] D. Collins, A. Zijdenbos, V. Kollokian, J. Sled, N. Kabani, C. Holmes, and A. Evans. Design and construction of a realistic digital brain phantom. IEEE Transactions on Medical Imaging, 17(3):463-468, 1998.

[4] H. Goldstein. Classical Mechanics. Addison-Wesley, Reading, MA, 1950.

[5] F. Kruggel and D. von Crammon. Alignment of magneticresonance brain datasets with the steretactical coordinate system. Medical Image Analysis, 3(2):175-185, 1999.

[6] R.-S. Kwan, A. Evans, and G. Pike. MRI simulation-based evaluation of image-processing and classification methods. IEEE Transactions on Medical Imaging, 18(11):1085-1097, 1999.

[7] Y. Liu, R. Collins, and W. Rothfus. Robust midsagittal plane extraction from normal and pathological 3D neuroradiology images. IEEE Transactions on Medical Imaging, 20(3):175192, 2001.

[8] J. Maintz and M. Viergever. A survey of medical image registration. Medical Image Analysis, 2(1):1-36, 1998.

[9] J. F. Mangin, V. Frouin, I. Bloch, J. Regis, and J. LopezKrahe. From 3D magnetic resonance images to structural representations of the cortex topography using topology preserving deformations. Journal of Mathematical Imaging and Vision, 5:297-318, 1995.

[10] G. Marola. On the detection of the axes of symmetry of symmetric and almost symmetric planar images. IEEE Transactions on Pattern Analysis and Machine Intelligence, 11:104108, 1989.

[11] T. Masuda, K. Yamamoto, and H. Yamada. Detection of partial symmetry using correlation with rotated-reflected images. Pattern Recognition, 26:1245-1253, 1993.

[12] P. Minovic, S. Ishikawa, and K. Kato. Symmetry identification of a 3d object represented by octree. IEEE Transactions on Pattern Analysis and Machine Intelligence, 15(5):507514, 1993.

[13] D. O'Mara and R. Owens. Measuring bilateral symmetry in digital images. In Proceedings of TENCON'96 IEEE Conference: Digital Signal Processing Applications, volume 1, pages 151-156, 1996.

[14] W. Press, S. Teukolsky, W. Vetterling, and B. Flannery. Numerical Recipes in C. 2nd Edition. Cambridge University Press, Cambridge, 1992.

[15] S. Prima, S. Ourselin, and N. Ayache. Computation of the Mid-Sagittal Plane in 3D Images of the Brain. In D. Vernon, editor, Sixth European Conference on Computer Vision, ECCV'2000, volume 1842-3 of Lecture Notes in Computer Science, pages 685-701, Dublin, Irlande, 2000. Springer.

[16] C. Sun and J. Sherrah. 3-D symmetry detection using the extended Gaussian image. IEEE Transactions on Pattern Analysis and Machine Intelligence, 19(2):164-168, 1997.

[17] J.-P. Thirion, S. Prima, G. Subsol, and N. Roberts. Statistical Analysis of Normal and Abnormal Dissymmetry in Volumetric Medical Images. Medical Image Analysis, 4(2):111121, 2000.

[18] H. Zabrodsky, S. Peleg, and D. Avnir. Symmetry as a continuous feature. IEEE Transactions on Pattern Analysis and Machine Intelligence, 17:1154-1166, 1995. 